\section{P1-47 AN INNOVATIVE CASE-SPOUSE CONTROL DESIGN IN PRACTICE: AN EXPERIENCE IN ESTIMATING SMOKING AND ALL CAUSE DEATHS IN CHINESE ADULTS}

doi:10.1136/jech.2011.142976c.40

J Jiang, * B Liu, F Sitas, J Li, X Zeng, W Han, X Zou, Y Wu, P Zhao. Department of Epidemiology and Biostatistics, Chinese Academy of Medical Sciences (CAMS), Peking Union medical College (PUMC), Beijing, China

Introduction We aimed to show that utilising information from a population-based, retrospective mortality survey for the main determinants of disease is feasible.

Methods During 1989-1991, a nationwide retrospective survey of mortality was conducted. To assess the effects of smoking on all cause mortality in Chinese adults, we used a new design within this survey. For all the deceased persons (cases) we sex-matched surviving spouses as controls. Smoking information for cases was provided by spouse or other informants, at the same time as providing their own smoking history. Results For all cause mortality, the results revealed that tobacco use was associated with $11.2 \%$ of total deaths in 1987 , and more than two-thirds of these excess deaths occurred between the ages of 50 and 74 years. Although life expectancies varied with region or sex, the years of life lost attributable to smoking was almost the same in each age group. Our study also confirmed that more than $50 \%$ of the sex difference in life expectancy was accounted for by smoking. With respect to the novel design, the results revealed consistency in the results using different control groups.

Conclusion This new case-spouse control design as an alternative for control selection in case-control studies is valid and feasible.

\section{P1-48 MATHEMATICAL MODELS TO INFORM THE DESIGN, ANALYSIS AND INTERPRETATION OF VACCINE TRIALS}

doi:10.1136/jech.2011.142976c.41

P Scott, ${ }^{*}$ N Low. Institute of Social and Preventive Medicine (ISPM), University of Bern, Bern, Switzerland

Background When planning vaccine trials or interpreting results, it is not always clear which outcome measure best estimates the effect of the vaccine and at which time point it should be measured. We used a mathematical model to explore questions arising from the analysis of pneumococcal nasopharyngeal carriage data from conjugate vaccine trials, in which the acquisition-rate ratio (ARR) is the desired effect measure. We determine the time at which the prevalence ratio $(\mathrm{PR})$ or prevalence $\mathrm{POR}$, which are usually available, provide the best approximation.

Methods We created a hypothetical randomised controlled trial using a dynamic compartmental model which incorporated carriage of vaccine-type Streptococcus pneumoniae in vaccinated and unvaccinated groups. ARR was incorporated explicitly, linking the acquisition rate in the vaccinated to the acquisition rate in the unvaccinated and was assumed not to change for 2 years. Prevalence ratios and prevalence ORs for carriage were plotted over time.

Results The PR approximates the ARR well, after an initial period in which the PR decreases from one and stabilises. The length of this period is determined by the duration of carriage. During the period when the PR approximates the ARR, the POR overestimates the effect of vaccination. This is less marked when carriage is rare.

Conclusions This model illustrates the behaviour of outcome measures for pneumococcal carriage in a simple setting and can be elaborated to explore more complex situations. Mathematical models provide opportunities to explore study designs and analysis methods for both planned and completed vaccine trials.

\section{P1-49 SPATIAL EPIDEMIOLOGICAL MAPPING FOR DISEASE PREVALENCE MANAGEMENT IN AN URBAN COMMUNITY: SWINE FLU PREVALENCE MANAGEMENT IN SOUTH OF TEHRAN, IRAN}

doi:10.1136/jech.2011.142976c.42

M M Sepehri, ${ }^{*}$ H Ehrami, B Teimourpour. Tarbiat Modares University, Tehran, Iran

Epidemiology investigation uses multidisciplinary approaches to help decision makers in healthcare to control and foretell the phenomena of disease prevalence. In this study, we have applied spatiotemporal analysis and mapping to improve swine flu prevalence management in south of Tehran, Iran. We present a new pattern to monitor the swine flu pandemic in Iran in a more effective way. In this research we gathered 900 suspicious records of H1N1 from south of Tehran. We used spatial data mining and spatiotemporal analysis method to create a specific final pattern for a potential swine flu pandemic management and recovery. GIS and data mining tools have been used to calculate and visualise the results. The results of this research can be used by health policy makers and administrators to guide mitigation policies to minimise possible spread of the disease into the general healthcare setting.

\section{P1-50 SHOULD HEART FAILURE BE CONSIDERED AS CATEGORICAL OR DIMENSIONAL?}

doi:10.1136/jech.2011.142976c.43

${ }^{1,2} \mathrm{M}$ Severo, ${ }^{*}{ }^{1,3} \mathrm{~A}$ Azevedo, ${ }^{1,4} \mathrm{R}$ Gaio. ${ }^{1}$ Department of Hygiene and Epidemiology, University of Porto Medical School, Porto, Portugal; ${ }^{2}$ Institute of Public Health of the University of Porto, Porto, Portugal; ${ }^{3}$ Heart Failure Clinic, Department of Internal Medicine, Hospital S. João, Porto, Portugal; ${ }^{4}$ Department of Mathematics, University of Porto Science School, Porto, Portugal

Introduction The categorical view dominates the traditional diagnostic approach to heart failure (HF) but ignores possible withinclass heterogeneity such as individual differences in severity.

Objective To assess if HF should be considered as categorical or dimensional, and to validate a novel scale of severity for clinical HF

Methods Cross-sectional evaluation of 1115 community participants aged $\geq 45$ years in 2006-2008. We considered items related to troubled breathing and fatigue (4 items), volume overload (6 items) and objective evidence of cardiac structural /functional abnormalities (3 items). Bayesian Information criteria from latent class analysis (LCA) and latent trait analysis (LTA) were used to assess if HF could be considered as categorical or dimensional. BNP values and American College of Cardiology (ACC)/ American Heart Association (AHA) stages of HF, classified by experienced clinicians with access to all data, were used to validate the scale.

Results Bayesian Information criteria suggested a 3-class solution for the LCA and a 2-factor solution for the LTA, with the best result being the last one. The first factor was associated with the items on troubled breathing/fatigue and cardiac abnormalities; the second factor was associated with the items about volume overload. The prevalence of $\mathrm{BNP} \geq 30 \mathrm{pg} / \mathrm{ml}, \mathrm{BNP} \geq 100 \mathrm{pg} / \mathrm{ml}$ and stage C/D of clinical HF was significantly higher in the group of individuals with high scores for all factors than in that of individuals with low scores.

Conclusions The use of latent models applied to HF provided evidence for considering HF as dimensional rather than categorical as traditionally considered. 